\title{
Prefibrillar Tau Oligomers in Mild Cognitive Impairment and Alzheimer's Disease
}

\author{
Elliott J. Mufson ${ }^{\mathrm{a}}$ Sarah Ward ${ }^{\mathrm{b}}$ Lester Binder ${ }^{\mathrm{b}}$ \\ ${ }^{a}$ Department of Neurological Sciences, Rush University Medical Center, and ${ }^{b}$ Department of Cell and \\ Molecular Biology, Feinberg School of Medicine, Northwestern University, Chicago, Ill., USA
}

\section{Key Words}

Alzheimer's disease · Fibrillar aggregates - Mild cognitive impairment . Oligomers · Tau

\begin{abstract}
Background: Alzheimer's disease (AD) is a progressive neurodegenerative disorder characterized by the accumulation of extracellular amyloid- $\beta$ peptide and intracellular tau. Here, we review data suggesting that prefibrillar tau oligomers mediate cognitive decline early in the disease. Objective: It was our aim to study the presence of tau-positive pretangle neurons and correlate findings with cognitive test scores. Methods: Pretangle antibodies (TOC1 and pS422) were applied to tissue containing cholinergic basal forebrain neurons from people who died with a premortem clinical diagnosis of no cognitive impairment, mild cognitive impairment and AD. Results: Data lend support to the concept that tau oligomers are the toxic form of tau, that non-fibillar tau relates to cognitive dysfunction and that the earliest pretangle pathology occurs in neuritic processes. Conclusions: Clinicopathological findings highlight the importance of studying tau modifications in neuronal soma and neuritic processes, which may be the earliest pathological lesions that correlate with cognitive status.

(c) 2013 S. Karger AG, Basel
\end{abstract}

(c) 2013 S. Karger AG, Basel

$1660-2854 / 13 / 0133-0151 \$ 38.00 / 0$
Alzheimer's disease (AD), the most common form of dementia, is clinically characterized by progressive cognitive impairment [1] and pathologically by extracellular amyloid- $\beta(A \beta)$ plaques and intracellular accumulation of the microtubule-associated protein tau into neurofibrillary tangles (NFTs) [2]. Unlike A $\beta$ plaques, the spatial and temporal progression of NFTs positively correlates with the progression of clinical symptoms [3], and NFT load correlates with neuronal cell loss and the severity of cognitive impairment in $\mathrm{AD}$ [4]. Tau is necessary for $\mathrm{A} \beta$-induced neurotoxicity in cell culture and transgenic mouse models $[2,5]$, occurs in tauopathies lacking $A \beta$ pathology [6], and tau gene mutations cause some forms of frontotemporal dementia [7]. In $\mathrm{AD}$, tau is hyperphosphorylated and undergoes conformational shifts resulting in its self-association into filamentous and nonfilamentous aggregates [8]. However, whether or not filamentous tau aggregates are neurotoxic remains controversial. Findings suggest that neuronal dysfunction precedes the formation of these insoluble fibrillar deposits, suggesting that earlier non-fibrillar tau aggregates may be neurotoxic [9].

The existence of prefibrillar aggregates of recombinant tau has been reported in in vitro assembly assays [9], and tau-positive pretangle neurons lacking NFTs

\section{KARGER}

E-Mail karger@karger.com

www.karger.com/ndd
Elliott J. Mufson, PhD

Alla V. and Solomon Jesmer Chair in Aging

Rush University Medical Center, 1735 West Harrison Street

Suite 300, Chicago, IL 60612 (USA)

E-Mail emufson@ rush.edu 
Fig. 1. Bright-field photomicrographs of nucleus basalis CBF neurons dual stained for pS422 (brown) and TOC1 (dark blue) in $\mathrm{NCI}(\mathbf{a}), \mathrm{MCI}(\mathbf{b})$ and $\mathrm{AD}(\mathbf{c}, \mathbf{d})$. Arrows indicate single pS422 neurons. Confocal laser dual immunofluoresence (e) showing TOC1 (green), pS422 (red) and merged images of CBF neurons in MCI. Scale bars $=50 \mu \mathrm{m}$. Colors refer to the online version only.

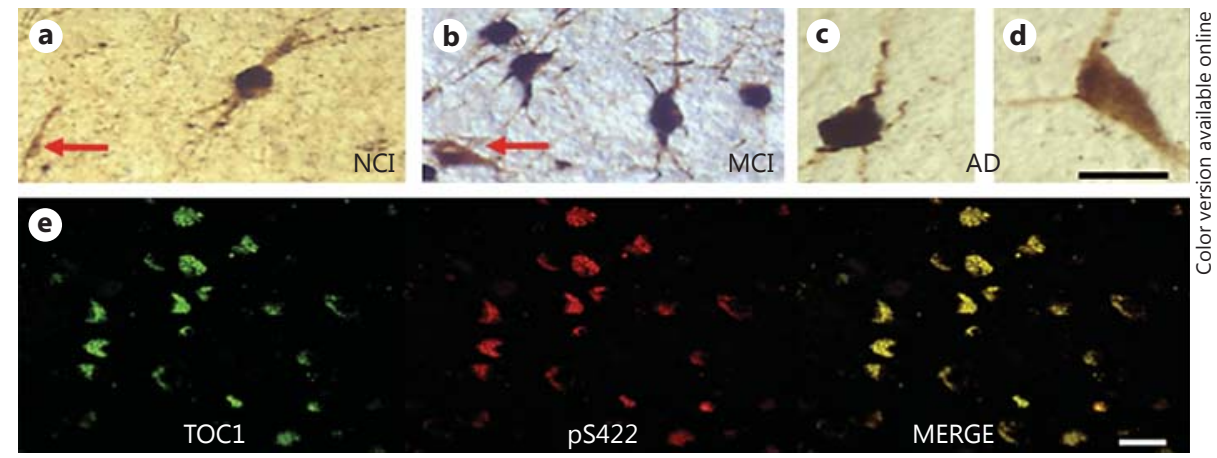

occur in the $\mathrm{AD}$ brain [9]. Recently, tau oligomers were found at a 4-fold higher concentration in $\mathrm{AD}$ than in healthy control brains [9]. Binder and colleagues [10] performed an in vitro crosslinking experiment demonstrating that tau filament formation is preceded by tau dimerization [10]. To validate the relevance of these findings to $\mathrm{AD}$, they generated a novel monoclonal antibody that selectively labels tau dimers and higher-order oligomers termed 'tau oligomeric complex 1' (TOC1) [10]. Therefore, in this report, an oligomer is defined as any aggregate of tau recognized by TOC1. Although oligomers may exist in multiple-size categories, virtually nothing is known about how many 'mers' make up tau oligomers. A recent atomic force microscopy study demonstrated that their oligomers were 40mers [11] but this has yet to be repeated by other groups and likely requires further investigation. TOC1 was shown to localize in pretangle neurons of aged controls but was more abundant in the superior, temporal and entorhinal cortex in $\mathrm{AD}[10]$. The presence of TOC1 immunoreactive inclusions in control cases (Braak stages I and II) suggests that dimer/oligomer formation is an early event in disease pathogenesis. Supporting this assertion, immunofluorescence in human tissue sections indicated that oligomerization closely associates with Ser422 phosphorylation (pS422), an early pathological event in AD [10]. Prefibrillar tau aggregates have been isolated from $\mathrm{AD}$ prefrontal cortex brain homogenates using atomic force microscopy, and in vitro studies indicate that the formation of similar granular oligomers precede paired helical filament formation [9]. TOC1 containing prefibrillar inclusions in $\mathrm{AD}$ lends support to in vitro tau aggregation and crosslinking findings performed by our group, suggesting that tau dimers/oligomers may be intermediates in the aggregation process that precede NFT formation in situ [10].
Due to difficulties in isolating and characterizing tau intermediates, direct evidence linking tau oligomers to neuronal dysfunction is limited. Recently, we utilized extruded axoplasm from the squid giant axon to study the effects of tau monomers and aggregates on axonal transport [9]. The addition of recombinant human tau monomers has no effect on transport when physiological concentrations of tau are used. However, addition of the same amounts of a mixture of recombinant tau oligomers plus filaments caused a selective inhibition of fast anterograde transport [9]. Furthermore, addition of the taubinding protein Hsp70 to the oligomer plus a filament mixture prevents fast anterograde transport inhibition in the squid axoplasm assay. Moreover, Hsp70 was demonstrated to bind to tau oligomers and not filaments, leading to the suggestion that oligomers are the primary cause of toxicity in this assay [12] as well as in AD and other tauopathies [6].

Only recently have studies been undertaken to determine whether neuronal non-fibrillar tau is related to cognitive function. A clinical pathological investigation by our group provides evidence for the onset of intraneuronal pretangle tau pathology indicated by the presence of pS422 within cholinergic basal forebrain (CBF) neurons in people with mild cognitive impairment (MCI) prior to frank CBF neuron loss, which we previously reported to correlate with $\mathrm{AD}$ neuropathological criteria and tests of cognitive function [13]. Interestingly, pS422 colabels the newly reported pretangle tau oligomeric marker TOC1 [9] within $\mathrm{CBF}$ neurons during the onset of $\mathrm{AD}$ (fig. 1), supporting the concept that pS422 accompanies tau oligomer formation. Clinical pathologic studies show that in the CBF, pS422 but not TauC3 (a marker for frank NFTs) positive CBF neurons correlate with cognitive decline in MCI [14]. Comparing the development of NFT pathology within the CBF with that of the medial tempo- 
ral lobe, using the appearance of the pretangle marker pS422 and the NFT marker, TauC3, indicated that NFT formation in $\mathrm{CBF}$ neurons is a more protracted process [9]. This suggests that NFT pathology develops at different rates depending on the site of pathology during dementia onset. An important question that remains to be investigated is the relationship between tau structures and toxic $A \beta$ oligomers. Interestingly, preliminary evidence from our group indicates that TOC1-positive tau oligomers are also present in progressive supranuclear palsy, cortical basal degeneration and chronic traumatic encephalopathy (data not shown), which do not display $\mathrm{A} \beta$ pathology, suggesting that toxic tau oligomers can form independently of $A \beta$. However, much work needs to be done to test this hypothesis.

In addition, we found that pS422+ neuropil thread length, i.e. tau pathology in dendrites and axons within the $\mathrm{CBF}$, increased in $\mathrm{MCI}$ and $\mathrm{AD}$, and this parameter correlated with both neuropathological criteria and cognitive test scores [14]. The magnitude of neuropil thread length compared to the number of pretangle and frank NFTs early in the disease suggests that pathogenesis in axonal and dendritic processes precedes that which occurs in the soma of CBF neurons, perhaps suggesting an alteration in axonal transport. These clinicopathological findings highlight the importance of studying not only tau modifications in neuron soma but changes to their neuritic processes, which may be the earliest site of tau pathology associated with cognitive decline.

\section{Acknowledgements}

This study was supported by NIA grants PO1AG14449 and PO1AG09466.

\section{References}

1 Cummings JL: Toward a molecular neuropsychiatry of neurodegenerative diseases. Ann Neurol 2003;54:147-154.

2 Rapoport M, Dawson HN, Binder LI, Vitek MP, Ferreira A: Tau is essential to beta-amyloid-induced neurotoxicity. Proc Natl Acad Sci USA 2002;99:6364-6369.

3 Braak H, Braak E: Neuropathological stageing of Alzheimer-related changes. Acta Neuropathol 1991;82:239-259.

-4 Arriagada PV, Marzloff K, Hyman BT: Distribution of Alzheimer-type pathologic changes in nondemented elderly individuals matches the pattern in Alzheimer's disease. Neurology 1992;42:1681-1688.

5 Roberson ED, Scearce-Levie K, Palop JJ, Yan F, Cheng IH, Wu T, Gerstein H, Yu GQ, Mucke L: Reducing endogenous tau ameliorates amyloid beta-induced deficits in an Alzheimer's disease mouse model. Science 2007; 316:750-754.
6 Feany MB, Dickson DW: Neurodegenerative disorders with extensive tau pathology: a comparative study and review. Ann Neurol 1996;40:139-148.

7 Poorkaj P, Grossman M, Steinbart E, Payami H, Sadovnick A, Nochlin D, Tabira T, Trojanowski JQ, Borson S, Galasko D, Reich S, Quinn B, Schellenberg G, Bird TD: Frequency of tau gene mutations in familial and sporadic cases of non-Alzheimer dementia. Arch Neurol 2001;58:383-387.

-8 Iqbal K, Liu F, Gong CX, Alonso Adel C, Grundke-Iqbal I: Mechanisms of tau-induced neurodegeneration. Acta Neuropathol 2009; 118:53-69.

9 Himmelstein DS, Ward SM, Lancia JK, Patterson KR, Binder LI: Tau as a therapeutic target in neurodegenerative disease. Pharmacol Ther 2012;136:8-22.

10 Patterson KR, Remmers C, Fu Y, Brooker S, Kanaan NM, Vana L, Ward S, Reyes JF, Philibert K, Glucksman MJ, Binder LI: Characterization of prefibrillar tau oligomers in vitro and in Alzheimer disease. J Biol Chem 2011; 286:23063-23076.
11 Sahara N, Maeda S, Takashima A: Tau oligomerization: a role for tau aggregation intermediates linked to neurodegeneration. Curr Alzheimer Res 2008;5:591-598.

12 Patterson KR, Ward SM, Combs B, Voss K, Kanaan NM, Morfini G, Brady ST, Gamblin TC, Binder LI: Heat shock protein 70 prevents both tau aggregation and the inhibitory effects of preexisting tau aggregates on fast axonal transport. Biochemistry 2011;50:1030010310.

13 Mufson EJ, Binder L, Counts SE, DeKosky ST, de Toledo-Morrell L, Ginsberg SD, Ikonomovic MD, Perez SE, Scheff SW: Mild cognitive impairment: pathology and mechanisms. Acta Neuropathol 2012;123:13-30.

14 Vana L, Kanaan NM, Ugwu IC, Wuu J, Mufson EJ, Binder LI: Progression of tau pathology in cholinergic basal forebrain neurons in mild cognitive impairment and Alzheimer's disease. Am J Pathol 2011;179:2533-2550. 